\title{
El costo de ignorar el signo de Carnett. Reporte de caso y revisión de la literatura
}

\section{The Cost Of Ignoring Carnett's Sign: A Case Report and Literature Review}

\author{
William Otero Regino, MD, ${ }^{1}$ Erika Martínez Rodríguez, MD, ${ }^{2}$ Adán Lúquez Mindiola, MD. ${ }^{3}$
}

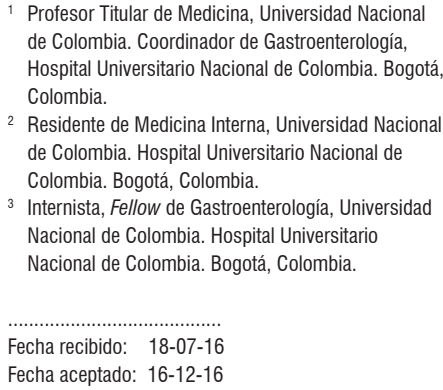

2 Residente de Medicina Interna, Universidad Nacional de Colombia. Hospital Universitario Nacional de Colombia. Bogotá, Colombia.

${ }^{3}$ Internista, Fellow de Gastroenterología, Universidad Nacional de Colombia. Hospital Universitario Nacional de Colombia. Bogotá, Colombia.

Fecha recibido: $\quad 18-07-16$ Fecha aceptado: 16-12-16

\begin{abstract}
Resumen
El dolor abdominal crónico es un motivo frecuente de consulta externa y de urgencias. Los médicos tradicionalmente consideran que el origen del mismo son las estructuras intraabdominales, incluido el tracto gastrointestinal. Rara vez tienen en cuenta la pared abdominal como la causa de la molestia y someten a los pacientes a numerosos e interminables procedimientos diagnósticos, como laparoscopias y cirugías, entre otros. Por lo menos el $50 \%$ de estos pacientes tiene dolor de la pared abdominal por lesión del nervio cutáneo anterior y el diagnóstico se hace identificando el signo de Carnett. Se presenta un caso típico con esta patología, cuyo enfoque inicial ilustra los errores y altos costos en el abordaje de esta patología.
\end{abstract}

\section{Palabras clave}

Dolor abdominal crónico, nervio cutáneo anterior, signo de Carnett, costos.

\begin{abstract}
Chronic abdominal pain is a frequent cause of outpatient and emergency visits. Doctors traditionally consider that its origin is in intra-abdominal structures, including the gastrointestinal tract. They rarely take into account the abdominal wall as a cause of discomfort and subject patients to numerous and endless diagnostic procedures, including laparoscopy and surgery. At least $50 \%$ of these patients have abdominal wall pain due to injuries to the anterior cutaneous vein the diagnosis of which is made by identifying Carnett's sign. A typical case of this pathology is here. The initial approach illustrates the errors and high costs that can be involved in the approach to this pathology.
\end{abstract}

\section{Key words}

Chronic abdominal pain, anterior cutaneous nerve, Carnett's sign, costs.

\section{INTRODUCCIÓN}

El dolor abdominal crónico tiene múltiples causas y en la práctica médica es un desafío para los médicos de cuidado primario, los especialistas y los profesionales de los hospitales de referencia (1-3). En gastroenterología, el 50\% de los pacientes tiene un dolor idiopático y en el 10\%-90\% de ellos se observa que el origen del mismo está en la estructuras de la pared abdominal. En cuanto a estas, la lesión o compresión del nervio cutáneo anterior es la etiología más frecuente (2-5). Sin embargo, los médicos usualmente consideran que la causa del mismo son las vísceras abdominales y rara vez piensan en la posibilidad de estudiar la pared abdominal $(1,3,5)$. Después de investigar las causas viscerales, los pacientes terminan con diagnóstico de enfermedad sicosomática, síndrome del intestino irritable (SII) o gastritis, entre otros, y reciben tratamiento con analgésicos, ansiolíticos, antidepresivos o benzodiacepinas, y no es 
infrecuente que sean remitidos a psiquiatría. En el presente artículo, publicamos un caso típico de la forma en que este síntoma es abordado por los médicos de cuidado primario y de los centros de referencia.

\section{PRESENTACIÓN DEL CASO CLÍNICO}

Paciente masculino de 58 años de edad, con múltiples consultas al servicio de urgencias en el último año por cuadro de dolor abdominal localizado en el hipocondrio y flanco izquierdos, sin otra sintomatología asociada. Tampoco había signos de compromiso orgánico. Antecedente de dislipidemia y tío con cáncer de colon. Por segunda vez fue hospitalizado durante la última consulta médica por persistencia del dolor abdominal. Los exámenes de químicas sanguíneas fueron normales, el uroanálisis y la ecografía abdominal fueron normales también, así como la tomografía axial computarizada (TAC). El servicio de cirugía general descartó abdomen agudo quirúrgico, solicitó endoscopia digestiva alta, cuyo resultado fue gastritis crónica. Por persistencia del dolor abdominal, solicitaron ecografía de la pared abdominal, que fue normal. Continuaron el estudio con colonoscopia, que también fue normal. Al cuarto día solicitaron valoración por gastroenterología. $\mathrm{Al}$ examen físico, fue evidente la presencia del signo de Carnett A y B (indicativo de dolor de la pared abdominal). Gastroenterología no encontró otras alteraciones durante el examen físico abdominal. Se infiltraron los sitios del dolor con lidocaína y el dolor desapareció inmediatamente. Tres horas después, al paciente se le dio salida.

Con base en las tarifas del SOAT de 2015 (Ministerio de Protección Social de Colombia. Manual tarifario SOAT, Decreto 2423 de 2006 - actualizado 2015), sin incluir los medicamentos, los costos aproximados de la última consulta del paciente al servicio de urgencias se muestran en la tabla 1.

\section{DISCUSIÓN}

Con el advenimiento de equipos y pruebas diagnósticas cada vez más modernos y eficientes, progresivamente se ha ido perdiendo el interés por el examen físico de los pacientes en los diferentes escenarios de la medicina clínica. Esta desatención está produciendo un desmesurado aumento en solicitud de ayudas diagnósticas que, como en este caso, hubieran sido obviadas con un adecuado examen físico. En pacientes con dolor abdominal crónico, este puede originarse en las estructuras de la pared abdominal, que incluyen la piel, el tejido celular subcutáneo, el peritoneo parietal, los músculos y los nervios cutáneos anteriores procedentes de las raíces dorsales de T7 a T12 (4, 5), siendo este último la principal causa del mismo.
Tabla 1. Costos aproximados de la atención del paciente (SOAT 2015)

\begin{tabular}{lcc}
\hline \multicolumn{1}{c}{ Servicios y ayudas diagnósticas } & SMDLV & \$COP \\
\hline Tomografía de abdomen total & 22 & 492500 \\
Ultrasonografía abdominal total & 6,7 & 145200 \\
Ultrasonografía de tejidos blandos y & 6,7 & 145200 \\
pared abdominal & & \\
Colonoscopia total & 10 & 214780 \\
Sedación para colonoscopia & 2,3 & 49600 \\
Esofagogastroduodenoscopia & 10 & 214780 \\
diagnóstica & & \\
Hemograma & 0,8 & 17800 \\
Uroanálisis & 0,53 & 11400 \\
Valoración de urgencias & 1,96 & 42300 \\
Valoración por especialista (cirugía & 1,5 & 32400 \\
general) & & \\
Valoración por especialista & 1,5 & 32400 \\
(gastroenterología) & & \\
Hospitalización en habitación bipersonal & 11 & $236900 \times 4$ días \\
& & 947600 \\
Sala de observación, urgencias III nivel & 3,6 & 77300 \\
Infiltración de la pared abdominal & 1,7 & 37800 \\
\hline Total costos & 80,3 & 2461060 \\
\hline
\end{tabular}

COP: pesos colombianos; SMDLV: salario mínimo diario legal vigente.

Los nervios cutáneos sensoriales que salen de las raíces nerviosas de T7-T12 se angulan 90 grados al pasar por el anillo fibrosos del borde lateral de los músculos rectos anteriores, y también tienen una angulación similar al llegar a la piel $(2,4)$. Estas angulaciones los hacen particularmente vulnerables a los traumatismos o atrapamientos, entre otros.

En 1977, Thompson y colaboradores concluyeron que con la identificación del signo de Carnett puede diferenciarse un cuadro de abdomen agudo por irritación del peritoneo visceral del dolor originado por compresión o atrapamiento del nervio cutáneo anterior, que es la principal causa de dolor crónico de la pared abdominal (DCPA) $(1,2)$. Con este breve examen físico, se evitan exámenes innecesarios y procedimientos dispendiosos, invasivos y demás costos que impactan económicamente a los servicios de salud $(1,2)$. El dolor abdominal es uno de los primeros tres motivos de consulta más frecuentes en los servicios de urgencias y consulta externa, con una incidencia aproximada del $8 \%$ en las diferentes series (3). De estos, el $30 \%$ corresponde a dolores crónicos de la pared abdominal $(3,4)$. En nuestro medio, Otero y colaboradores encontraron que casi el $50 \%$ de los pacientes con dolor abdominal crónico (más de cuatro semanas) tenía DCPA, identificado con el signo de Carnett positivo (2). Estos pacientes igualmente habían sido sometidos a múltiples exámenes, medi- 
camentos, incluso cirugías y hospitalizaciones. La tercera parte de ellos tenía diagnóstico final de SII.

Después de descartar una causa quirúrgica del dolor abdominal, hay múltiples posibilidades etiológicas. Recientemente se ha encontrado que aplicando un cuestionario muy sencillo se logra identificar un gran número de pacientes con dolor crónico de la pared abdominal, quienes previamente habían sido diagnosticados con SII (5). Constanza y colaboradores (6), en un estudio de cinco años en pacientes con dolor abdominal crónico recurrente, encontraron SII en el 16,3\% y DCPA en el $7,8 \%$.

El signo de Carnett fue descrito en 1927 por J.B. Carnett, un obstetra inglés $(7,8)$. Inmediatamente después de su reconocimiento, se utilizó para diagnosticar lo que él y sus colaboradores denominaron neuralgia intercostal, nombre que hacía referencia a la irritación radicular de las últimas siete raíces dorsales y/o de la primera lumbar (origen de la inervación de la pared abdominal anterior). Esta causaba cuadros de dolor abdominal capaces de simular enfermedades como apendicitis, alteraciones renales o de la vesícula, o alteraciones del útero y sus anexos, pero que en realidad correspondían a una hipersensibilidad de la pared abdominal, que en la gran mayoría de los casos resolvía después de la infiltración del sitio doloroso, con anestésicos locales $(8,9)$.

En publicaciones, tanto nacionales (2) como internacionales $(5,6,9,10)$, se ha demostrado que la no identificación de este signo retrasa el diagnóstico y aumenta considerablemente los costos debido a la solicitud de exámenes innecesarios, así como por la ejecución de cirugías no indicadas.

A los pacientes con este cuadro clínico usualmente les solicitan múltiples exámenes que incluyen ecografía y TAC abdominal, endoscopias gastrointestinales e incluso procedimientos quirúrgicos innecesarios como apendicetomía, colecistectomía o laparoscopia diagnóstica, entre otros. La ejecución de estos diferentes procedimientos representa un gasto innecesario para el sistema de salud, ya que no permiten llegar a un diagnóstico correcto y el paciente continúa con deterioro en su calidad de vida $(1,3,5)$. Thompson y colaboradores (11) compararon el costo promedio de la evaluación de pacientes con dolor abdominal crónico, en quienes el dolor de la pared abdominal era un diagnóstico de exclusión, y encontraron gastos superiores a los $\$ 6000$ dólares por paciente, que finalmente lograban notable mejoría después de infiltrar la pared abdominal con anestésicos locales. Así mismo, Constanza y colaboradores (6) documentaron que el costo anual de cada paciente con dolor abdominal crónico fue de aproximadamente $\$ 11$ 000 dólares, comparado con $\$ 540$ dólares anuales cuando el diagnóstico de dolor crónico de la pared abdominal (DACPA) se hacía con base en el signo de Carnett.

En nuestro medio, Otero y colaboradores (2) reportaron un total de 1168 consultas previas al diagnóstico de
DACPA, con 278 procedimientos y 14 hospitalizaciones en un grupo de 91 pacientes, calculando un costo de $\$ 18$ 000 dólares ( 54 millones COP) con base en las tarifas del Seguro Social de 2001, por la atención del total de pacientes, con un costo promedio de \$200 dólares (\$594 500 COP) por paciente, sin incluir los costos de medicamentos, exámenes de laboratorio, como tampoco el ausentismo laboral (2). En un estudio (6) se documentó que sólo el 4\% de los médicos encuestados consideró la pared abdominal como el origen del cuadro de dolor abdominal crónico.

En este artículo publicamos el caso de un paciente con DACPA, de aproximadamente dos años de evolución, quien durante su última valoración por dolor abdominal fue hospitalizado y el costo de dicha hospitalización fue de aproximadamente $\$ 1000$ dólares ( $\$ 3000000 \mathrm{COP}$ ), equivalentes a 80 días de salario de un trabajador con el ingreso mensual mínimo permitido por la legislación colombiana. ¡El cuadro de dolor se resolvió con un procedimiento que tomó menos de 10 minutos y con un costo que no superó los $\$ 15$ dólares!

Cuando los médicos aprenden a identificar este importante signo y se familiarizan con él, los beneficios son evidentes $(2,6,10)$.

La forma de identificar el signo de Carnett es la siguiente $(2,8)$ : con el paciente en decúbito dorsal, sucesivamente se presionan diferentes puntos en el borde externo de los músculos rectos anteriores, en busca de un punto doloroso, con un área no mayor de $1,5 \mathrm{~cm}^{2}$ (figuras 1 y 2). Ocasionalmente sobre el sitio del dolor hay alodinia (sensación dolorosa anormal al tacto, que es un estímulo que normalmente no produce dolor) (1).

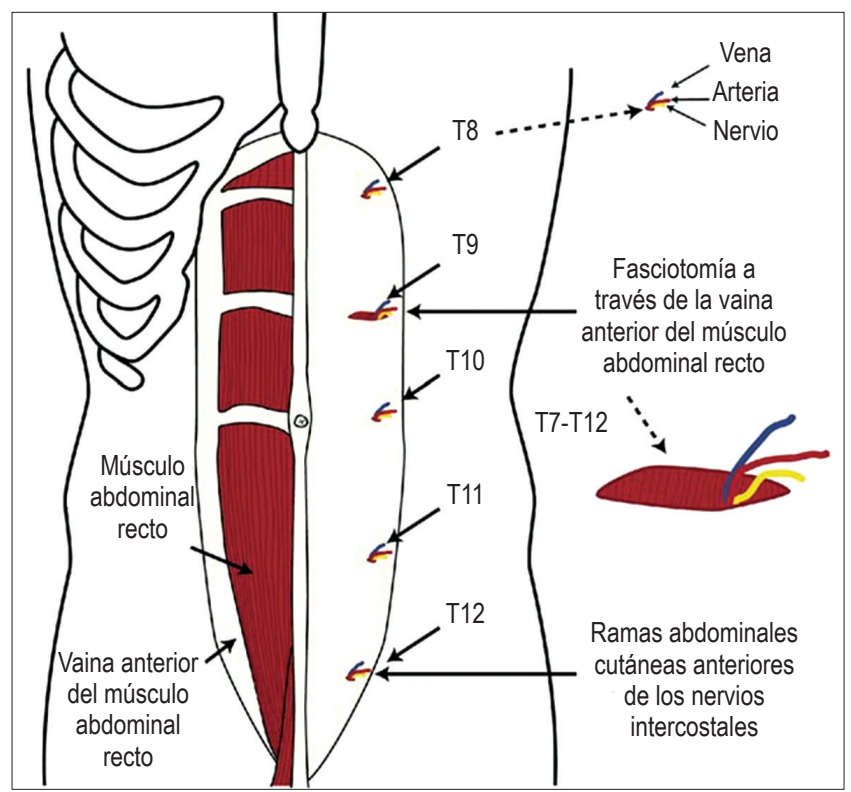

Figura 1. Salida de los nervios cutáneos anteriores. Modificado de referencia 4. 


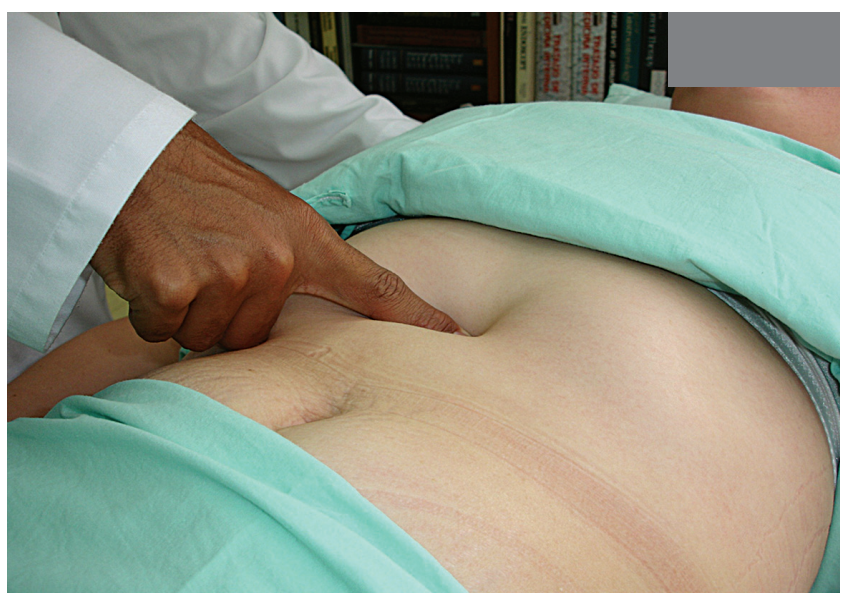

Figura 2. Identificación del sitio del dolor. Carnett A positivo.

Cuando se identifica el sitio del dolor, se mantiene la presión con el dedo (nosotros preferimos hacerlo con el pulgar de la mano derecha); se le pide al paciente que trate de sentarse lentamente, con lo cual se tensan los músculos abdominales. Si al tensar los músculos abdominales el dolor se mantiene sin cambios o aumenta de intensidad, el signo es positivo e indica que se origina en el nervio cutáneo anterior (figura 3). La positividad de esta segunda parte es la parte B del signo de Carnett. Si el dolor desaparece, se considera que este se origina en las estructuras viscerales.

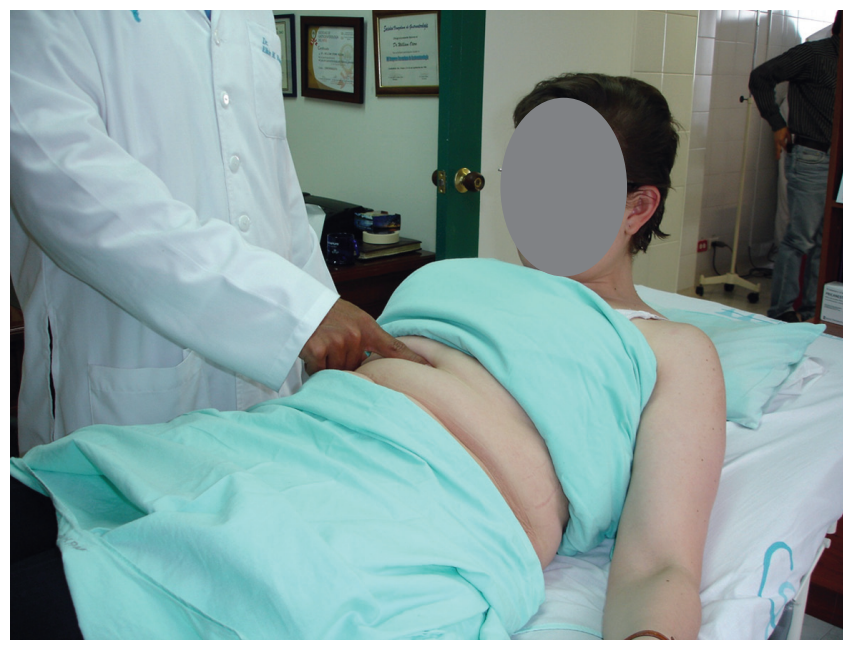

Figura 3. Signo de Carnett positivo (parte B).

El mecanismo del aumento del dolor, en casos de originarse en la pared, es que por la contracción de los músculos rectos anteriores, la presión del dedo en el sitio del dolor es más intensa, y en caso de que se originara en estructuras viscerales, el músculo contraído las protege de la presión del dedo y entonces desaparece $(2,8)$. Otra forma de tensar los músculos abdominales puede ser elevando los miem- bros inferiores o tosiendo; en ambas circunstancias debe mantenerse el dedo sobre el sitio del dolor. Este último método es una modificación que nosotros hemos hecho a la descripción original.

Cuando el punto doloroso se identifica con las características mencionadas, la positividad del mismo tiene sensibilidad y especificidad del $88 \%$ y del $97 \%$, respectivamente (12).

En nuestro servicio, el protocolo de infiltración es el siguiente (2): inicialmente se identifica el sitio del dolor y una vez encontrado, se marca haciendo una cruz, con un esfero (figura 4).

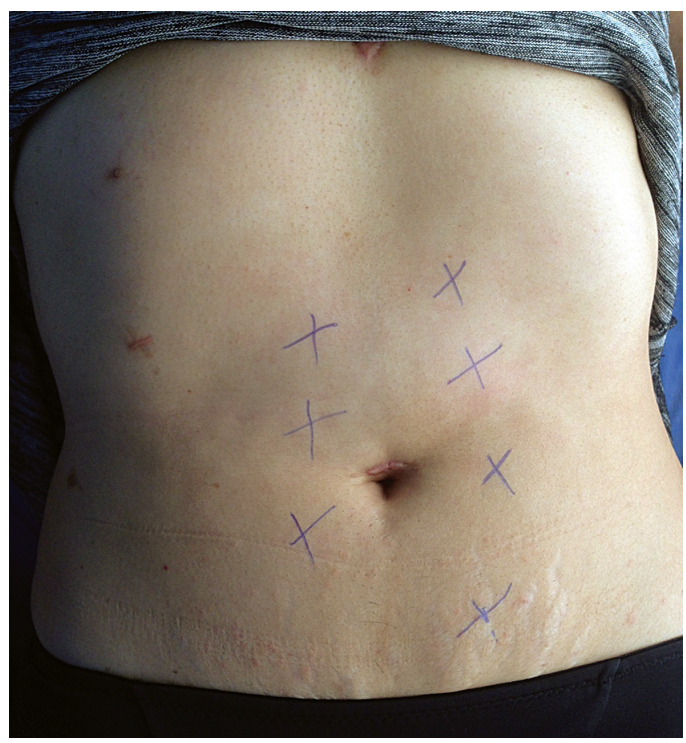

Figura 4. Marcación de los puntos dolorosos con signo de Carnett A y B positivos.

Se hace limpieza con alcohol y se infiltra con 1-2 mL, lidocaína al 2\% sin epinefrina (2). Dependiendo del grosor del panículo adiposo, se elige una aguja de insulina (calibre 27 de media pulgada) para las personas delgadas, o una aguja calibre 23 de 1 pulgada o una aguja calibre 21 de 1,5 pulgadas (la aguja convencional que traen las jeringas desechable). Las agujas mencionadas se montan en una jeringa de 5-10 mL (figuras 5, 6 y 7 ).

En una sesión pueden infiltrarse varios puntos, con la recomendación de no administrar más de $10 \mathrm{~mL}$, dado el riesgo de eventos adversos cardiovasculares. El paciente siempre debe firmar un consentimiento informado específico para este procedimiento. Si es menor de edad, el consentimiento deberá firmarlo cualquiera de sus padres o su representante legal. El objetivo es alcanzar el nervio cutáneo anterior o su vecindad (2). Después de la inyección, el dolor desaparece en más del $80 \%$ y con dos o más inyecciones se alcanza hasta un 91\% (2). La ineficacia del tratamiento con el anestésico local puede deberse a diver- 
sos factores (1), tales como dificultad para ver la llegada al sitio del dolor, por obesidad, agujas cortas, mala técnica o diagnóstico incorrecto.

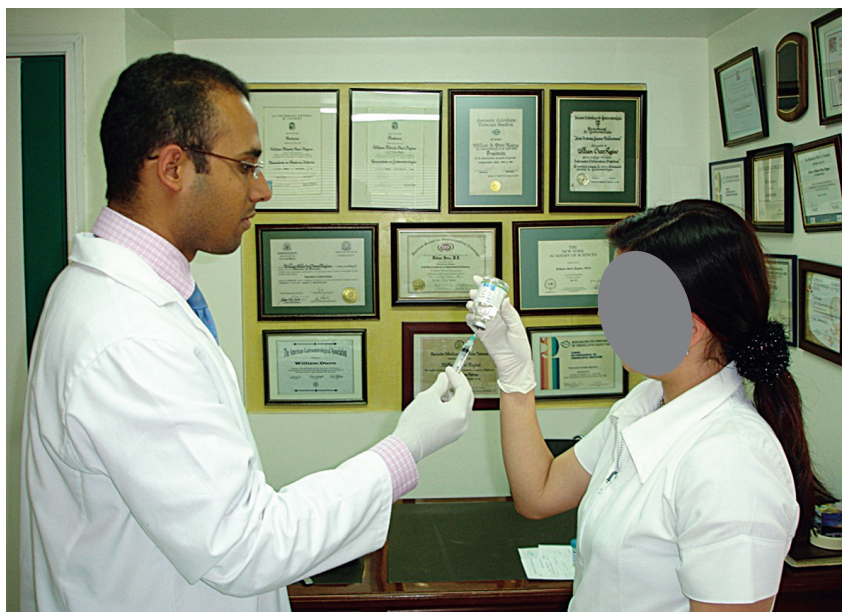

Figura 5. Envasando lidocaína al 2\% sin epinefrina.

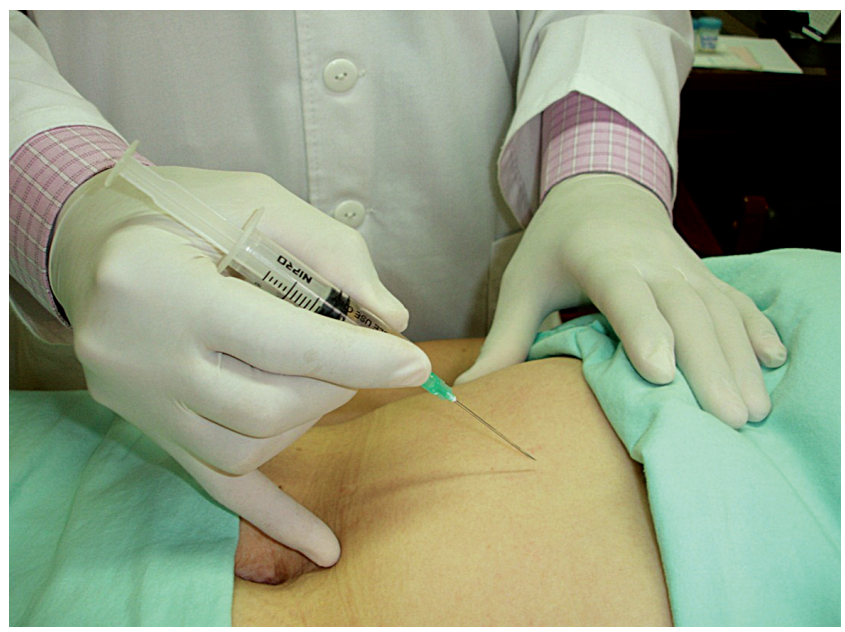

Figura 6. Eligiendo el sitio de aplicación de la lidocaína.

Ante un cuadro doloroso con las características mencionadas, no son necesarias otras pruebas confirmatorias. En un reciente ensayo clínico (11), se demostró que la infiltración con lidocaína del sitio del dolor tiene eficacia superior a la infiltración con solución salina como placebo $(p=0,007)$.

También se ha documentado que en pacientes con dolor pélvico crónico y signo de Carnett positivo, la infiltración semanal de la pared abdominal con lidocaína al 1\% + epinefrina, durante 5 a 6 semanas, produce desaparición del dolor en el 77\% de las pacientes y mejoría en el 93\% (13). Estos resultados destacan la importancia de detectar el signo de Carnett en este tipo de pacientes y que la infiltración del sitio del dolor puede ser un procedimiento efectivo en ginecología y obstetricia (11). En niños también se ha encontrado esta patología y se ha demostrado que el tratamiento local es eficaz (10).

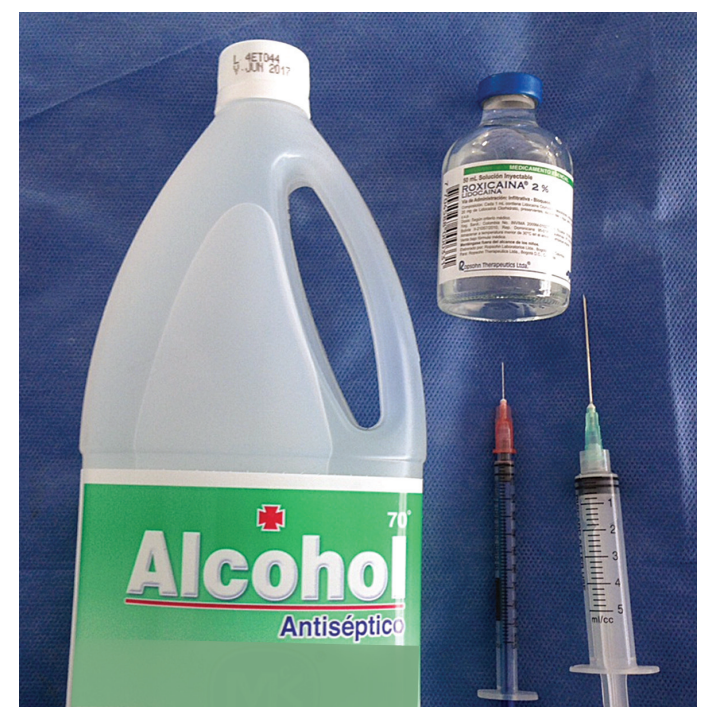

Figura 7. "Kit de infiltración".

No obstante la demostrada mejoría del dolor postinfiltración con lidocaína, se ha encontrado que el estrés crónico y las alteraciones sicológicas y las enfermedades siquiátricas concomitantes pueden predecir una respuesta menos favorable al tratamiento, aunque el signo de Carnett sea positivo $(14,15)$. Estos casos específicos están influidos por síndromes de somatización o por problemas psicosomáticos que requieren un abordaje multidisciplinario $(14,15)$.

Tradicionalmente el signo de Carnett se ha utilizado en la evaluación de pacientes con dolor abdominal crónico; sin embargo, también ha demostrado su utilidad en pacientes con dolor abdominal agudo $(16,17)$. En el estudio de Thomson, solo 1 de 24 pacientes con signo de Carnett positivo tenía una causa visceral del dolor agudo, y Gray y colaboradores (17) encontraron apendicitis en 5 de 158 $(3,1 \%)$ pacientes con el signo positivo. Cuando hay abdomen agudo y peritonismo, este signo no tiene utilidad (18).

En casos de dolor intratable, se ha utilizado la cirugía local como el tratamiento de elección (19). En tales casos, se hace disección local del nervio cutáneo con sección del mismo, con ligadura del paquete vascular acompañante. Otras patologías, como la herniación de los discos intervertebrales a nivel dorsal ( $75 \%$ por debajo de $\mathrm{T} 7$ ), pueden ser causales de dolor crónico de la pared abdominal, con una prevalencia que se considera baja. Sin embargo, estudios como el de Lara y colaboradores encontraron una prevalencia de hernia discal torácica de hasta un $30 \%$ en un grupo pequeño de pacientes con dolor abdominal crónico y Carnett positivo; no obstante, casi el $90 \%$ de los pacientes de este estudio reportó dolor abdominal constante aso- 
ciado con dolor dorsal, que se exacerbaba con el esfuerzo. La localización más común fue hacia los flancos, lo que podría ayudar a orientar aquellos casos de dolor abdominal crónico que no mejoran con infiltración de anestésicos y que podrían requerir de estudio con resonancia magnética de columna dorsal (13).

Con frecuencia no puede determinarse el origen de la alteración del nervio cutáneo anterior. Sin embargo, diversas circunstancias de la vida cotidiana pueden lastimarlo. Eventos como la tos crónica, los traumas abdominales, las prendas de vestir ajustadas, el vómito recurrente, la distensión abdominal o los movimientos repetitivos de aumento de la presión abdominal pueden producir lesión del nervio cutáneo anterior, que terminará en dolor abdominal crónico (13). Otras causas son el atrapamiento del nervio cutáneo anterior del abdomen (ACNES), causado por el aumento de la presión abdominal $(2,8,10)$, el pinzamiento por una cicatriz quirúrgica, los hematomas de la vaina del recto o las hernias incisionales $(18,19)$.

\section{CONCLUSIÓN}

En pacientes con dolor abdominal crónico, al igual que en todos los escenarios de la medicina, es fundamental una historia clínica detallada y un examen físico completo, y no sustituirlos por la tecnología, ya que la exploración de signos clínicos como el de Carnett es fundamental para descartar un gran número de diagnósticos diferenciales y evitar múltiples procedimientos adicionales que resulten engorrosos e infructuosos, a la vez que malgastan los fondos del sistema de salud $(1,2,6,11,20,21)$ y que no permiten identificar y solucionar la enfermedad.

\section{Financiación}

Los costos de este trabajo fueron asumidos en su totalidad por los autores.

\section{Conflicto de intereses}

ElDr. Otero ha dictado conferencias y ha recibido honorarios de Abbott-Lafrancol, Tecnofarma, laboratorios La-Santé, Procaps y laboratrios Takeda. La Dra. Erika Martínez y el Dr. Adán Lúquez no manifestaron conflicto de intereses.

\section{Agradecimientos}

Los autores agradecen a Alejandra Figueroa, enfermera asistente, y a Liliana Oino, ingeniera biomédica, su interés en el seguimiento y asignación de citas de los pacientes con esta patología en la Unidad de Gastroenterología de la Clínica Fundadores.

\section{REFERENCIAS}

1. Koop H, Koprdova S, Schürman C. Chronic abdominal wall pain. Dtsch Arztebl Int. 2016;113(4):51-7.

2. Otero W, Ruiz X, Otero E, et al. Dolor de la pared abdominal: una entidad olvidada con gran impacto en la práctica médica. Rev Col Gastroenterol. 2007;22:261-71.

3. Tolba R, Shroll J, Kanu A, et al. The epidemiology of chronic abdominal pain. En: Kapural L. Chronic abdominal pain. An evidence-based, comprehensive guide to clinical management. New York: Springer Science-Business Media; 2015. p. 13-24.

4. Lidsetmo RO, Stulberg J. Chronic abdominal wall pain-A diagnostic challenge for the surgeon. Am J Surg. 2009; 198:129-34.

5. Van Assen T, de Jager-Kievit JW, Scheltinga MR, et al. Chronic abdominal wall pain misdiagnosed as functional abdominal pain. J Am Board Fam Med. 2014;26(6):738-44.

6. Constanza CD, Longstreth GF, Liu AL. Chronic abdominal wall pain: clinical feature, health care costs and long term outcome. Clin Gastroenterol Hepatol. 2004;2(5):395-99.

7. Carnett JB. Intercostal neuralgia as a cause of abdominal pain and tenderness. Surg Gynecol Obstet. 1926;42:625-32.

8. Carnett JB, Bates W. The treatment of intercostal neuralgia of the abdominal wall. Ann Surg. 1933;98(5):820-9.

9. Thomson WHF, Dawes RFH, Carter SSC. Abdominal wall tenderness: a useful sign in chronic abdominal pain. Br J Sur. $1991 ; 78(2): 223-5$.

10. Akhnikh S, De Korte N, De Winter P. Anterior cutaneous nerve entrapment syndrome (ACNES): The forgotten diagnosis. Eur J Pediatrics. 2014;173:445-9.

11. Thomson C, Goodman R, Rowe WA, et al. Abdominal wall syndrome: a costly diagnosis of exclusion. Gastroenterology. $2001 ; 120(5): 637 \mathrm{~A}$.

12. Takada T, Ikusaka M, Ohira Y, et al. Diagnostic usefulness of carnett's test in psychogenic abdominal pain. Int Med. 2011;50(3):213-7.

13. Boelens OBA, Scheltinga MF, Houterman S, et al. Randomized clinical trials on trigger point infiltration with lidocaine to diagnose anterior cutaneous nerve entrapment syndrome. Br J Surg. 2013;100:217-21.

14. Lara FJ, Berges AF, Quesada JQ, et al. Thoracic disk herniation, a not infrequent cause of chronic abdominal pain. Int Surg. 2012;97(1):27-33.

15. Feurle GE. Abdominal wall pain--classification, diagnosis and treatment suggestions. Wien Klin Wocht. 2007;119(2122):633-8.

16. Thomson H, Francis DMA. Abdominal-wall tenderness: a useful sign in the acute abdomen. Lancet. 1977;310(8047):1053-4.

17. Gray DW, Seabrook G, Dixon JM, et al. Is abdominal wall tenderness a useful sign in the diagnosis of non-specific abdominal pain? Ann R Coll Surg Engl. 1988;70:233-4.

18. Rivero M, Moreira V, Riesco JM, et al. Dolor originado en la pared abdominal: una alternativa diagnóstica olvidada. Gastroenterol Hepatol. 2007;30(4):244-50. 
19. van Assen T, Boelens OB, van Eerten PV. Long term success rate after an anterior neurectomy in patients with abdominal nerve entrapment syndrome. Surgery. 2015; 157:137-43.

20. Alas A, Kim DS, Ogunyemi DAA. Do trigger point injections with buffered lidocaine improve pelvic pain of myo- fascial origin - a retrospective study. J Min Inv Gynecol. 2012;19(Suppl.1):S28-32.

21. Abdominal wall tenderness test: could Carnett cut costs? Lancet. 1991;337 (8750):1134. 